\title{
Directionality for nuclear recoils in a liquid argon Time Projection Chamber
}

\author{
Bianca Bottino ${ }^{a, b, 1, *}$ \\ ${ }^{a}$ Princeton University \\ Jadwin Hall, Washington Road, Princeton, New Jersey, USA \\ ${ }^{b}$ Istituto Nazionale di Fisica Nucleare, Sez. di Genova \\ Via Dodecaneso 33, Genoa, Italy \\ E-mail: bianca.bottino@ge.infn.it
}

Liquid argon (LAr) is one of the most promising targets for the search of WIMP-like dark matter. LAr dual-phase time projection chamber (LAr TPC) is a leading technology, able to detect both the scintillation and ionization signal. The correlation in the two signal channels provides a possible handle to measure the recoil direction of the nuclei: if confirmed, this would allow inferring the incident direction of potential dark matter candidates. Previous work from SCENE resulted in a hint of the existence of a directional effect, which can potentially pave the way for a tonne scale directional WIMP search with LAr TPC. To validate this hypothesis, we conducted the Recoil Directionality (ReD) experiment to measure this correlation in $70 \mathrm{keV}$ nuclear recoils to the highest precision. The ReD TPC was carefully calibrated and then irradiated with a neutron beam at the INFN Laboratori Nazionali del Sud, Catania, Italy. A model based on directional modulation in charge recombination was developed to explain the correlation. In this contribution, we briefely summarize the theoretical model, and then we focus on the description of the experimental setup and the preliminary results from data analysis.

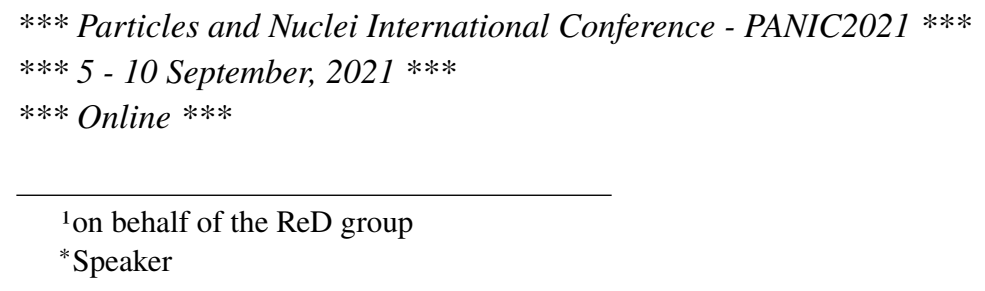




\section{The goal: investigate directional sensitivity}

The DarkSide collaboration has demonstrated, using the DarkSide-50 detector [1], extraordinary background rejection capability and it is now preparing for the construction of the DarkSide-20k detector [2, 3]. Like its predecessor, DarkSide-20k will be housed at the INFN Gran Sasso underground laboratory (LNGS), and it is expected to attain a WIMP-nucleon cross section 90\% C.L. exclusion sensitivity of $7.4 \times 10^{-48} \mathrm{~cm}^{2}$ for a WIMP mass of $1 \mathrm{TeV} / \mathrm{c}^{2}$ in a $200 \mathrm{t} \cdot \mathrm{yr}$ run. DarkSide-20k is designed to limit the number of instrumental background interactions to $<0.1$ events during the whole exposure, so that a positive claim could be made with as few WIMP events as possible. The only irreducible background source will be due to the coherent elastic neutrino-nucleus scattering interactions, which is a particularly challenging background because it can mimic an authentic WIMP signal. One possible feature that could be exploited to discriminate $v$-induced nuclear recoils from WIMP-induced events is the sensitivity to the direction of the incoming particle. The expected WIMP events rate, assuming the Standard Halo Model, is strongly anisotropic, due to the motion of the solar system within our galaxy, in particular we expect that dark matter induced recoil distribution is pointing opposite to the Cygnus constellation direction. So having a dark matter detector with sensitivity to the direction on the recoil would allow to identify any signal with the dark matter galactic halo and hence to make an unambiguous dark matter discovery. The ReD-Recoil Directionality project wants to verify whether it is possible to operate a directional detector made with a two-phase liquid argon Time Projection Chamber (TPC). Hints of such directional phenomena have already been observed by the SCENE experiment [4]. The main goal of the experiment is to prove the directionality sensitivity, in the WIMP-Ar scattering energy range, exploiting the dependence of columnar recombination on the alignment of the recoil momentum with the electric field present inside the detector.

\section{The TPC working principle}

The TPC consists of an active volume filled with liquid, which is immersed in a uniform electric field. This electric field drifts ionization electrons towards a gaseous region on top of the detector, where charges are accelerated to stimulate light production by electro-luminescence. Photo-detectors collect both the prompt scintillation light produced in liquid (S1), and the delayed electro-luminescence light (S2) generated in gas, allowing for energy measurement and 3D vertex reconstruction [1]. Only a fraction of the ionized electrons produced in liquid can avoid the recombination process, due to the presence of the electric field that drifts them away from the positive ions. Some columnar recombination models suppose that the recombination fraction could depend on the angle between the recoil direction and the drift electric field [5, 6]. If recoil track is almost parallel to the electric field, the free electron must pass through almost the whole column during the drift, maximizing the recombination probability. On the other hand if recoil track is perpendicular to the electric field, electrons will drift for a short distance inside the ionized particles cloud and then pass to the region of neutral atoms, reducing the recombination probability. 


\section{The ReD TPC design and performance}

The ReD TPC has several key characteristics in common with the future DarkSide-20k experiment, including some mechanics, but on a smaller scale. The key novel feature is a readout system based on cryogenic Silicon Photomultipliers (SiPMs), which are employed and operated continuously for the first time in an argon TPC. Over the course of 6 months, the ReD TPC was commissioned and characterised under various operating conditions using $\gamma$-ray and neutron sources, demonstrating remarkable stability of the optical sensors and reproducibility of the results. The ReD TPC has an active volume of $5 \times 5 \times 6 \mathrm{~cm}^{3}$ and all the walls are made in by $3 \mathrm{M}$ reflector film, sandwiched by two $1.5 \mathrm{~mm}$ acrylic plates. Also the anode and the cathode, placed at the top and the bottom of the TPC, are made in acrylic, coated with a $25 \mathrm{~nm}$ ITO conductive layer on both sides. In order to convert the $128 \mathrm{~nm}$ photons emitted by argon to visible light, the whole internal surface is coated with wavelength shifter (TPB, tetraphenyl butadiene). The SiPMs, developed by FBK Foundation for the DarkSide Collaboration, are grouped in two $5 \times 5 \mathrm{~cm}^{2}$ tiles, each containing 24 SiPMs. The tile located at the top of the TPC has a 24-channel readout board, in order to read each SiPM individually. In the bottom tile the SiPMs are summed in group of six, so that the front end board has a 4-channel readout. The TPC response was studied by using an external ${ }^{241} \mathrm{Am}$ source, which emits monoenergetic $\gamma \mathrm{s}$ of $59.54 \mathrm{keV}$. The response to scintillation light (S1) of the detector was studied by operating it filled of liquid argon only, in the so-called single phase mode, without any electric field. The light yield, obtained by the ${ }^{241} \mathrm{Am}$ peak, accounting for cross talk and after pulse in the SiPMs is $9.80 \pm 0.13 \mathrm{PE} / \mathrm{keV}_{e e}$. The position of the energy peak of ${ }^{241} \mathrm{Am}$ is found to be reproducible within $2 \%$. By operating the TPC in dual-phase mode it was possible to study the $\mathrm{S} 2 / \mathrm{S} 1$ ratio, which is an important parameter in order to distinguish electron recoil events (ER), from nuclear recoils (NR). In figure 1 the $\mathrm{S} 2 / \mathrm{S} 1$ ratio is shown as a function of $\mathrm{S} 1$ for all single-scatter events with a prompt $\mathrm{S} 1$ and $\mathrm{S} 2$ signals, as well as for events with a neutron-like-induced NR signal. The band due to NR is clearly separated from the ER one for values above 200 photoelectrons (PE). Moreover the detector shows a good uniformity in the S2/S1 response, that is a key parameter for the performance of the TPC. From the S1 vs S2 yield anti-correlation, due to the different ratio of recombined and drifted electrons at different drift fields, the following experimental gains [4] have been found: a scintillation gain $\mathrm{g} 1=\left(0.195 \pm 0.018_{\text {stat }+ \text { sys }}\right) \mathrm{PE} /$ photon and an amplification gain

$\mathrm{g} 2=\left(20.7 \pm 1.6_{\text {stat }+ \text { sys }}\right) \mathrm{PE} / \mathrm{e}^{-}$. All the technical details about the ReD TPC and its performance can be found in [7].

\section{The full setup and the first data}

The strategy on which the full setup is based is to irradiate the ReD TPC with neutrons of known energy and direction. The neutrons are generated through the $\mathrm{p}(7 \mathrm{Li}, \mathrm{n}) 7 \mathrm{Be}$ reaction with the 15 MV Tandem accelerator, at the Laboratori Nazionali del Sud (LNS), in Catania. They can produce parallel or orthogonal nuclear recoils, with respect to the drift field, then after the scattering they are detected by a neutron spectrometer that - given the closed kinematics - allows the study of the signal as a function of the scattering angle. The dedicated neutron spectrometer is constituted by an array of nine 3-inch liquid scintillator neutron detectors (LScis). The primary ${ }^{7} \mathrm{Li}$ energy and the positions of the TPC and of the LScis are tuned such to select nuclear recoils of about $70 \mathrm{keV}$ 


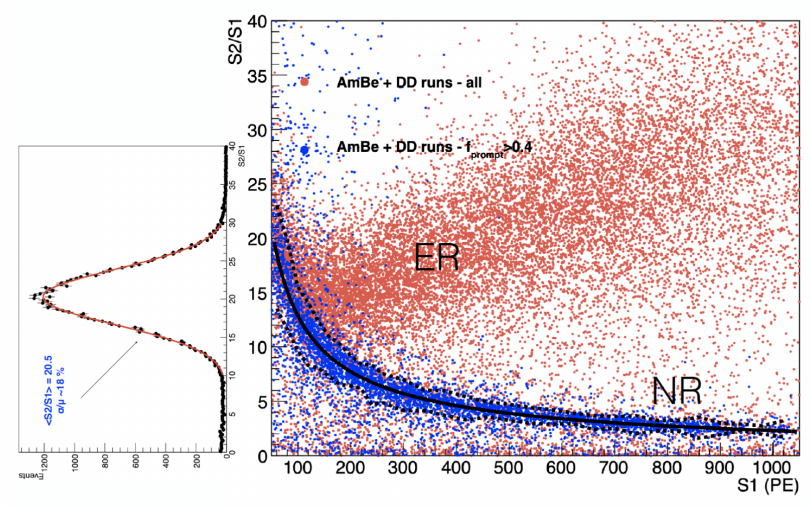

Figure 1: Distribution of NRs (blue points) compared to all events (red points) in the S2/S1 vs. S1 plane for the $\mathrm{AmBe}+\mathrm{DD}$ combined dataset. The black curve represents the most probable values of S2/S1 as a function of energy and the dashed lines are the $90 \%$ confidence level range.

in the TPC. Neutrons are tagged individually by detecting the associated 7Be nuclei by means of a $\Delta \mathrm{E} / \mathrm{E}$ telescope of silicon detectors $(\mathrm{Si})$, installed in the scattering chamber. Events of interest are selected as triple coincidence between Si, TPC and LSci. The event sample is further cleaned by the identification of ${ }^{7} \mathrm{Be}$ in the Si telescope, the timing and the pulse shape discrimination, both in the TPC and LSci. The whole setup was mounted, tested and a two-weeks beam run has been performed in February 2020. In conclusion, the whole experiment was successfully validated and triple coincidence events were identified, the next step will be to perform high statistics runs.

\section{Conclusions}

The ReD experiment has the objective to investigate the sensitivity to directionality in the energy range of interest for WIMP dark matter searches. For this purpose the group built and fully characterised a small double-phase argon TPC, featuring innovative readout by cryogenic SiPMs. Using $\gamma$-ray and neutron sources it was possible to measure the single photoelectron response, the light yield, and the scintillation and ionisation gains. The performances of the TPC were considered satisfactory in order to reach the scientific goal of the experiment. We therefore moved on to a phase of integration and testing of the entire system. The first calibration data are promising, but the analysis is still ongoing.

\section{References}

[1] P. Agnes et al., [DarkSide Collaboration], Phys. Lett. B 743, 456, (2015)

[2] P. Agnes et al., [DarkSide Collaboration], Phys. Rev. D 93, 081101, (2016)

[3] P. Agnes et al., [DarkSide Collaboration], Phys. Rev. D 98, 102006, (2018)

[4] H. Cao et al., Phys. Rev. D 91, 092007, (2015)

[5] J. B. Birks et al., Proc. Phys. Soc. A 64, 874, (1951) 
[6] V. Cataudella et al., JINST 12 (12), P12002, (2017)

[7] P. Agnes et al., Eur. Phys. J. C 81, 1014, (2021) 